\title{
émulations
}

\section{Des trajectoires familiales liées aux conditions du retour}

\section{Le cas des migrant·e·s rentré·e·s dans les DOM}

\author{
Marine Haddad
}

Émulations - Revue de sciences sociales 2020, n³4, «Transnationaliser le retour. Vers une révision du regard sur les migrations de retour contemporaines ».

\section{Article disponible à l'adresse suivante}

https://ojs.uclouvain.be/index.php/emulations/article/view/haddad

\section{Pour citer cet article}

Marine Haddad, « Des trajectoires familiales liées aux conditions du retour. Le cas des migrant·e.s rentré.e.s dans les DOM », Émulations, $n^{\circ} 34$, Mise en ligne le 7 octobre 2020.

DOI : 10.14428/emulations.034.02

Distribution électronique : Université catholique de Louvain (Belgique) : ojs.uclouvain.be

(C) Cet article est mis à disposition selon les termes de la Licence Creative Commons Attribution, Pas d'Utilisation Commerciale 4.0 International. http://creativecommons.org/licenses/by-nc/4.0/

Éditeur : Émulations - Revue de sciences sociales / Presses universitaires de Louvain https://ojs.uclouvain.be/index.php/emulations

ISSN électronique : 1784-5734

PUL PRESSES

UNIVERSITAIRES 


\title{
Des trajectoires familiales liées aux conditions du retour
}

\author{
Le cas des migrant·e·s rentré·e·s dans les DOM
}

Marine Haddad ${ }^{1}$

\begin{abstract}
[Résumé] Cet article étudie les liens entre les trajectoires familiales des migrant·e.s de retour dans les départements d'outre-mer (Martinique, Guadeloupe, Guyane, la Réunion) et les conditions de leur retour. À partir de l'enquête Migrations, Famille et Vieillissement et d'analyses de séquences, il dresse une typologie des trajectoires de cohabitation, de mise en couple et de parentalité de ces populations. Cinq types de trajectoires sont mis en valeur : l'entrée rapide dans la parentalité déclinée selon trois configurations conjugales, le départ tardif du domicile parental et la décohabitation rapide non suivie de mise en couple ou naissance d'enfants. Des régressions analysent ensuite l'effet des retours sur les trajectoires. Pour les femmes, le retour retarde la naissance d'enfants ou l'union et favorise les ruptures, surtout en cas de contraintes ou difficultés. Pour les hommes, le retour accélère au contraire ces étapes. Ces analyses soulignent ainsi l'organisation genrée des calendriers migratoires et familiaux, dans un rapport différencié à la contrainte.
\end{abstract}

Mots-clés : migration de retour, outre-mer, famille, analyse de séquences.

Linking family trajectories and return conditions: the case of return migrants in the French Overseas Departments

[Abstract] This paper investigates the links between the family trajectories of return migrants in the French overseas departments (Martinique, Guadeloupe, French Guiana, Reunion Island) and the conditions of their return. Based on the Migration, Family and Ageing survey and sequence analysis, it draws up a typology of the trajectories of cohabitation, relationship and parenthood of these populations. It highlights five types of trajectories: rapid entry into parenthood with three possible conjugal patterns, late departure from the parental home and rapid decohabitation without subsequent mating or parenthood. Regressions then assess the effect returns have on trajectories. For women, returning delays childbearing or partnering and encourages the break-ups, especially in the case of constraints or difficulties. For men, on the contrary, returning accelerates these events. These analyses underline the gendered organization of migration and family calendars, in a differentiated relationship to constraint.

Keywords: return migration, French overseas, family, sequence analysis.

Les Antilles, la Guyane et la Réunion sont des espaces marqués par d'importants mouvements migratoires, qui structurent leur développement et leur traitement politique. Si les mobilités géographiques ont toujours fait partie du quotidien des populations d'outre-mer, les migrations vers la métropole ont connu un essor massif après la départementalisation, en 1946, et la mise en place de politiques incitatives, dès la fin des années 1950 (Anselin, 1990 ; Constant, 1987). Le chômage et le sous-emploi élevés dans les départements d'outre-mer (DOM) ont justifié la création de vastes dispositifs enca-

${ }^{1}$ Chercheure, INED, France. 
drant la migration vers la métropole, tels que le Bureau pour la migration des DOM (1963-1981), remplacé par l'Agence nationale pour l'insertion et la promotion des travailleurs d'outre-mer (1982-2006), puis l'Agence de l'outre-mer pour la mobilité (à partir de 2006). Ces politiques migratoires se sont appuyées sur une vision associant la forte croissance démographique de ces régions aux difficultés socioéconomiques rencontrées (Domenach, Picouet, 1992), tout en répondant à un besoin de main-d'œuvre peu qualifiée en métropole jusqu'à la fin des années 1970 (Condon, Ogden, 1991). Depuis les années 1980, les départs sont plus fréquents chez les jeunes les plus dotés en ressources économiques ou scolaires (Temporal, Marie, Bernard, 2011), mais ils sont toujours mis en avant comme un outil de lutte contre le chômage (Ihaddadene, 2017). Pour saisir l'effet de ces départs sur les DOM, il faut tenir compte de la dimension transrégionale de l'expérience des ultramarin·e·s : les liens économiques, affectifs ou culturels maintenus avec le DOM de départ (Condon, 1996), mais aussi la circularité potentielle des mobilités géographiques (Marie, Rallu, 2004). En effet, après quelques mois ${ }^{2}$ ou plusieurs décennies, les migrant·e·s sont susceptibles de rentrer dans leur département de naissance. L'expérience accumulée en métropole affecte alors leurs conditions de vie une fois de retour. Dans une perspective relationnelle, le retour de ces migrant.e.s au profil spécifique est aussi susceptible d'avoir une incidence sur les trajectoires des ultramarin·e.s qui n'ont pas migré, d'autant qu'elles et ils représentent une part importante de la population des DOM : en 2010, un quart des personnes nées dans les DOM et y habitant avait connu une migration, principalement vers la France métropolitaine3. Cet article étudie l'hétérogénéité de la population des migrant·ess de retour dans les DOM à la lumière de leurs trajectoires familiales et résidentielles.

Un nombre croissant de travaux se penchent sur les migrations circulaires, se focalisant sur les déterminants socioéconomiques des mobilités pour analyser les trajectoires de retour au prisme des capitaux accumulés en migration (De Coulon, Piracha, 2005 ; Skeldon, 2012). En revanche, les liens entre configurations familiales et trajectoires de retour demeurent sous-étudiés. La dimension familiale et résidentielle des histoires de vie est pourtant susceptible de jouer un rôle important dans les trajectoires de retour: directement, en déterminant des opportunités et contraintes qui ralentissent ou accélèrent le retour ; indirectement, en modalisant les parcours professionnels qui déterminent les conditions du retour. Une importante littérature analyse les effets réciproques liant migration et sphère familiale (Hondagneu-Sotelo, Avila, 1997 ; Hugo, 1981 ; Massey et al., 1990 ; Olwig, 2007), mais elle considère davantage les départs que les retours (Potter, Conway, Phillips, 2005). Approcher les trajectoires de retour au prisme des configurations familiales, à la fois structurantes et structurées par les départs vers la métropole, apporte donc un éclairage nouveau sur ces migrations spécifiques.

Du fait de la citoyenneté française des ultramarin·e·s, aucune barrière légale ne limite leurs mobilités vers la France métropolitaine. En revanche, elles et ils font face à des

${ }^{2}$ On considère qu'un séjour en dehors du DOM relève de la migration à partir d'une durée de six mois.

${ }^{3}$ Source : enquête Migrations, Famille et Vieillissement (INED, 2010). 
coûts et difficultés liés à l'éloignement géographique des DOM, ainsi qu'à des processus de catégorisation raciale (Brinbaum, Safi, Simon, 2012 ; Haddad, 2018). Ce positionnement entre migrations internes et internationales fait des mobilités DOM-métropole un cas unique pour comprendre les dynamiques migratoires contemporaines. Il permet de questionner la pertinence de la focale transnationale dans un contexte postcolonial (Meléndez, 2017). Les configurations familiales sont un vecteur structurant des migrations depuis les DOM (Attias-Donfut, Lapierre, 1997 ; Condon, Byron, 2007). De l'inquiétude publique liée à une forte croissance des populations ultramarines dans les années 1960 à l'influence des réseaux migratoires sur l'insertion des nouveaux arrivants, en passant par la plasticité de l'organisation des relations familiales, la famille et la capacité des ultramarin·e.s à « faire famille » entre deux territoires est au cœur de la question des migrations DOM-métropole. On peut donc faire l'hypothèse que la sphère familiale et son organisation au fil du temps sont non seulement liées à la migration vers la France métropolitaine, mais également aux conditions de retour dans les DOM.

Pour tester cette hypothèse, cet article étudie la diversité des trajectoires des migrant·e·s ultramarin.e.s de retour dans leur département de naissance (Martinique, Guadeloupe, Guyane, la Réunion). Il tient compte des profils socioéconomiques des migrant·e·s, mais également de leurs histoires de vie sur le plan familial et résidentiel. À partir des données de l'enquête Migrations, Famille et Vieillissement (MFV, INED, 2010), cette recherche se focalise sur les populations des DOM de plus de 35 ans ayant connu une expérience migratoire d'au moins six mois, le plus souvent en France métropolitaine. En mettant de côté les plus jeunes, cette focale met surtout en valeur des mécanismes concernant les migrations plus anciennes. L'analyse est menée en deux temps : d'abord, à l'aide de méthodes d'analyse de séquences, une typologie des trajectoires familiales que les migrant·ess de retour d'au moins 36 ans ont connues entre leurs 15 et 35 ans est construite ; puis, la relation entre ces types de trajectoires et les conditions de retour est évaluée à l'aide de modèles de régression. Les résultats montrent de quelle manière le retour affecte les calendriers familiaux selon des mécanismes de genre, favorisant les unions et naissances d'enfants pour les hommes, les retardant ou les brisant pour les femmes.

\section{Les conditions du retour dans l'enquête MFV}

MFV est une enquête réalisée par l'Institut national d'études démographiques entre 2009 et 2010 15770 habitant.e.s de Guadeloupe, Martinique, Guyane et la Réunion, de 18 à 79 ans, ont été interrogé·e.s et des informations détaillées ont été recueillies sur les expériences migratoires. L'échantillonnage des migrant·e·s de retour a été possible grâce aux données des enquêtes Emploi menées dans les DOM. Dans cette étude, on se focalise sur les personnes nées dans le DOM ayant vécu en dehors du DOM durant au moins six mois consécutifs, tel-le·s que sont défini-e.s les migrant·e·s de retour dans l'enquête. L'enquête fournit des informations détaillées sur leurs migrations, notamment

\footnotetext{
${ }^{4}$ Enquête réalisée avec le concours de l'INSEE, sous la responsabilité de Claude-Valentin Marie.
} 
les dates de leurs départs et retours pour leur premier et leur dernier séjour migratoire. On ne considère, parmi ces migrant·e.s de retour, que les personnes âgées de plus de 35 ans au moment de l'enquête, afin de pouvoir comparer leurs trajectoires familiales entre les âges de 15 et 35 ans - intervalle durant lequel la majorité des mobilités ont lieu (figures 1 et 2), ainsi que de nombreuses transitions comme le départ du domicile ou la naissance d'enfants. L'échantillon ainsi réduit compte 4015 personnes. Pour maintenir des effectifs suffisants, on réunit les données des quatre DOM.

Pour saisir la diversité des retours, cet article s'appuie sur deux indicateurs disponibles dans l'enquête : le fait de percevoir le retour comme un choix ou une contrainte et le fait d'avoir rencontré des difficultés dans les mois qui ont suivi le retour. La distribution de ces indicateurs parmi les migrant·e.s de retour met en valeur des situations contrastées (tableau 1). Si une majorité associe son retour à un choix et n'a pas fait l'expérience de difficultés, près d'un quart s'est senti contraint de revenir et plus d'un quart a rencontré des difficultés. De plus, les femmes associent plus souvent leur retour à une contrainte ( $24 \%$ contre $20 \%$ des hommes) et déclarent plus souvent avoir rencontré des difficultés (31 \% contre $24 \%$ des hommes). Pour mieux saisir ces écarts, on étudie leurs liens avec les trajectoires familiales, comprises comme séquences d'événements telles que le départ du domicile parental, la mise en couple ou encore la naissance des enfants.

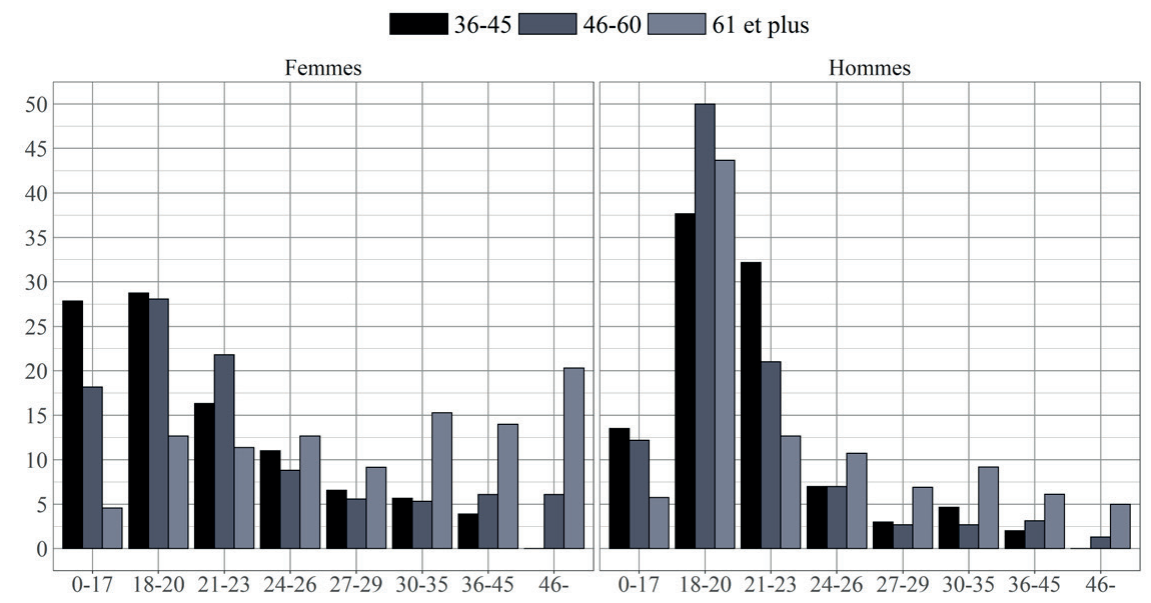

Figure 1 : Distribution des ÂGeS Au PREMIER déPART SELON L'ÂGE LORS DE L'ENQUÊTE ET LE SEXE (\%)

(POUR LES PERSONNES QUI ONT CONNU PLUS D'UNE MIGRATION, SEULES LES DATES DE LA PREMIÈrE MIGRATION SONT ICI PRISES EN COMPTE.)

Source : MFV (INED, 2010) | Intervalles de confiance à $95 \%$ entre crochets | Champ : personnes âgées d'au moins 36 ans, nées dans les DOM et ayant vécu au moins six mois hors de leur DOM de naissance 


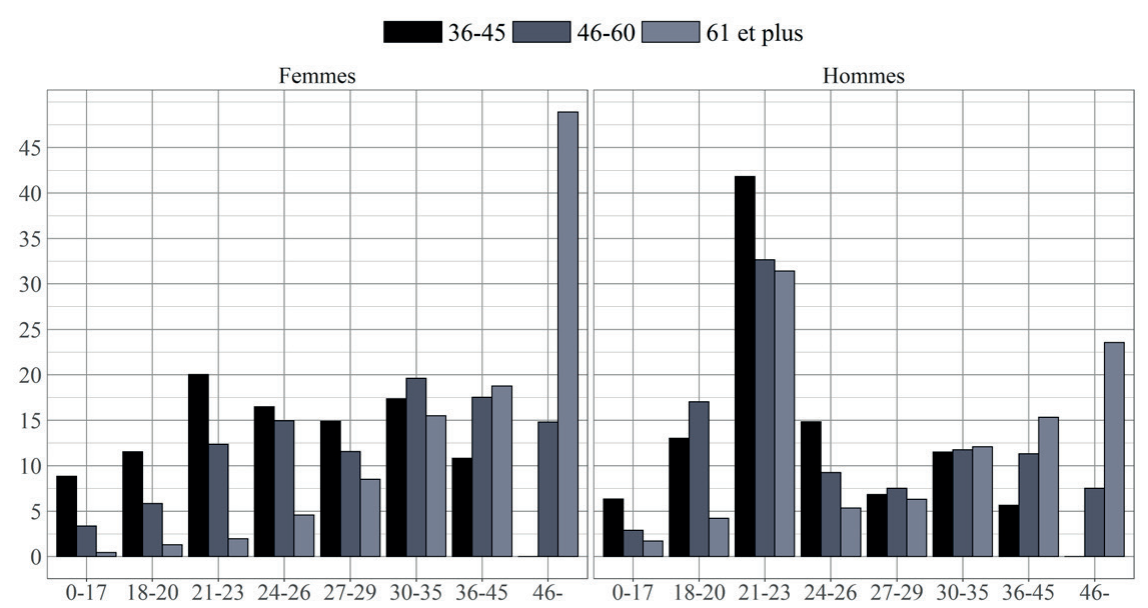

Figure 2 : Distribution des ÂGes au premier RETOUR SELON L'ÂGE LORS DE L'ENQuête et Le SEXe (\%)

(Pour LES PERSONNES QUI ONT CONNU PLUS D'UNE MIGRATION, SEULES LES DATES DE LA PREMIÈre MIGRATION SONT ICI PRISES EN COMPTE.)

Source : MFV (INED, 2010) | Intervalles de confiance à $95 \%$ entre crochets | Champ : personnes âgées d'au moins 36 ans, nées dans les DOM et ayant vécu au moins six mois hors de leur DOM de naissance

Tableau 1 : Diversité des profils de retour dans les DOM selon le sexe (\%)

\begin{tabular}{|l|c|c|c|}
\hline Question & Réponse & Hommes & Femmes \\
\hline $\begin{array}{l}\text { Votre décision de } \\
\text { retour dans le } \\
\text { département a-t-elle } \\
\text { reposé principale- } \\
\text { ment sur... }\end{array}$ & une contrainte & 20,0 & 24,1 \\
\cline { 2 - 4 } & un choix & 80,0 & 75,9 \\
\hline $\begin{array}{l}\text { À votre retour dans } \\
\text { le département, } \\
\begin{array}{l}\text { avez-vous rencontré } \\
\text { des difficultés } \\
\text { particulières? }\end{array}\end{array}$ & Oui & 23,9 & 31,5 \\
\cline { 2 - 4 } & Non & 76,1 & 68,5 \\
\hline
\end{tabular}

Source : MFV (INED, 2010) | Champ : personnes âgées d'au moins 36 ans, nées dans les DOM et ayant vécu au moins six mois hors de leur DOM de naissance

\section{Des trajectoires familiales à trois dimensions}

On considère l'évolution des configurations familiales au fil du temps, et pas seulement leur état à un moment donné, en construisant une typologie des séquences d'événements liés aux configurations familiales entre 15 et 35 ans. Ces séquences sont fondées sur trois dimensions : le couple, les enfants et le mode de cohabitation. On utilise les variables portant sur : la date où l'individu quitte le logement familial, les dates de ses unions et séparations, les dates de cohabitation et décohabitation avec les compagnons ou compagnes, les dates de naissance de ses enfants éventuels. Le croisement de ces variables donne lieu à seize configurations possibles ; les plus rares sont regroupées 
ensemble, ce qui aboutit à une variable catégorielle à neuf modalités. Les modalités sont les suivantes :

- cohabite avec ses parents, non mariéee, n’a pas d'enfant ;

- cohabite avec ses parents, non mariéee, a un ou plusieurs enfants ;

- a quitté le domicile parental, non marié·e, n’a pas d'enfant ;

- a quitté le domicile parental, non mariée, a un ou plusieurs enfants ;

- cohabite avec un·e concubin·e, non mariéee, n’a pas d'enfant ;

- cohabite avec un·e concubin·e, non marié·e, a un ou plusieurs enfants ;

- cohabite avec un·e époux·se, n’a pas d'enfant ;

- cohabite avec un·e époux·se, a un ou plusieurs enfants ;

- $\quad$ autre (cohabitation avec ses parents et un·e époux·se, mariage décohabitant, etc.).

Les trajectoires familiales correspondent alors à l'évolution des valeurs prises par cette variable au cours du temps pour chaque individu et les méthodes d'analyse des séquences permettent de construire une typologie de ces trajectoires. De leurs 15 ans à 35 ans, les individus connaissent une séquence de vingt et un états, correspondant aux configurations familiales vécues chacune de ces vingt et une années. En séparant les analyses pour les hommes et les femmes, une typologie de ces séquences est construite en combinant les méthodes d'Optimal Matching Analysis (OMA) et de classification hiérarchique ${ }^{5}$. Les analyses aboutissent à une partition en cinq classes, pour les femmes comme pour les hommes ${ }^{6}$. Comme les classifications obtenues pour les deux sexes sont similaires et par souci de synthèse, elles sont présentées conjointement. Les effectifs et la fréquence de chaque classe varient au sein et entre les populations étudiées (tableau 2). Le nombre de personnes dans chaque classe par sexe n'est pas suffisant pour analyser de manière robuste la typologie en distinguant les DOM, néanmoins cette distribution varie peu selon la région dans notre échantillon (les écarts les plus importants sont observés pour les sous-échantillons aux effectifs les plus faibles, suggérant qu'ils viennent davantage de la petite taille du sous-échantillon que de réelles différences). Observer les trajectoires les plus fréquentes dans chaque classe permet de caractériser les classes 7 (figures 3 et 4 ).

\footnotetext{
${ }_{5}^{5}$ L'OMA mesure la dissemblance entre les séquences : une fonction de distance attribue un coût à chaque opération à effectuer sur les éléments pour que deux séquences deviennent identiques (inversion, insertion, délétion), puis les distances entre chaque paire de séquences de l'échantillon sont regroupées dans une matrice (MacIndoe, Abbott, 2004 ; Robette, Thibault, 2008). Une fois la matrice de distance obtenue, la méthode de classification hiérarchique lui est appliquée pour obtenir une typologie des séquences. La partition maximise la distance entre les classes et minimise la distance à l'intérieur des groupes. Les classes sont agrégées selon la méthode de Ward.

${ }^{6}$ On détermine le nombre de classe à l'aide du " critère du coude », c'est-à-dire le nombre de classes à partir duquel on observe un décrochage dans la perte de variance inter-classe.

${ }^{7}$ En complément, la distribution des configurations familiales à chaque âge est représentée dans les figures $\mathrm{A} 1$ et $\mathrm{A} 2$ en annexe.
} 
Tableau 2 : Distribution des classes de trajectoires familiales parmi les femmes et les hommes

\begin{tabular}{|c|c|c|c|c|c|c|}
\hline & \multicolumn{3}{|c|}{ Femmes } & \multicolumn{3}{|c|}{ Hommes } \\
\hline & Classe & $\mathrm{N}$ & $\%$ & Classe & $\mathrm{N}$ & $\%$ \\
\hline $\begin{array}{l}\text { Parentalité } \\
\text { hors union } \\
\text { cohabi- } \\
\text { tante }\end{array}$ & 1 & 335 & 17,8 & 1 & 192 & 9,0 \\
\hline $\begin{array}{l}\text { Parentalité } \\
\text { au sein du } \\
\text { mariage }\end{array}$ & 2 & 676 & 36,0 & 2 & 933 & 43,7 \\
\hline $\begin{array}{l}\text { Parentalité } \\
\text { au sein du } \\
\text { concubi- } \\
\text { nage }\end{array}$ & 3 & 163 & 8,7 & 3 & 286 & 13,4 \\
\hline $\begin{array}{l}\text { Départs } \\
\text { tardifs du } \\
\text { domicile } \\
\text { parental }\end{array}$ & 4 & 311 & 16,6 & 4 & 300 & 14,0 \\
\hline $\begin{array}{l}\text { Décohabi- } \\
\text { tation } \\
\text { sans } \\
\text { couple ni } \\
\text { enfant }\end{array}$ & 5 & 394 & 21,0 & 5 & 425 & 19,9 \\
\hline & & 1879 & 100,0 & & 2136 & 100,0 \\
\hline
\end{tabular}

Source : MFV (INED, 2010) | Champ : personnes âgées d'au moins 36 ans, nées dans les DOM et ayant vécu au moins six mois hors de leur DOM de naissance 

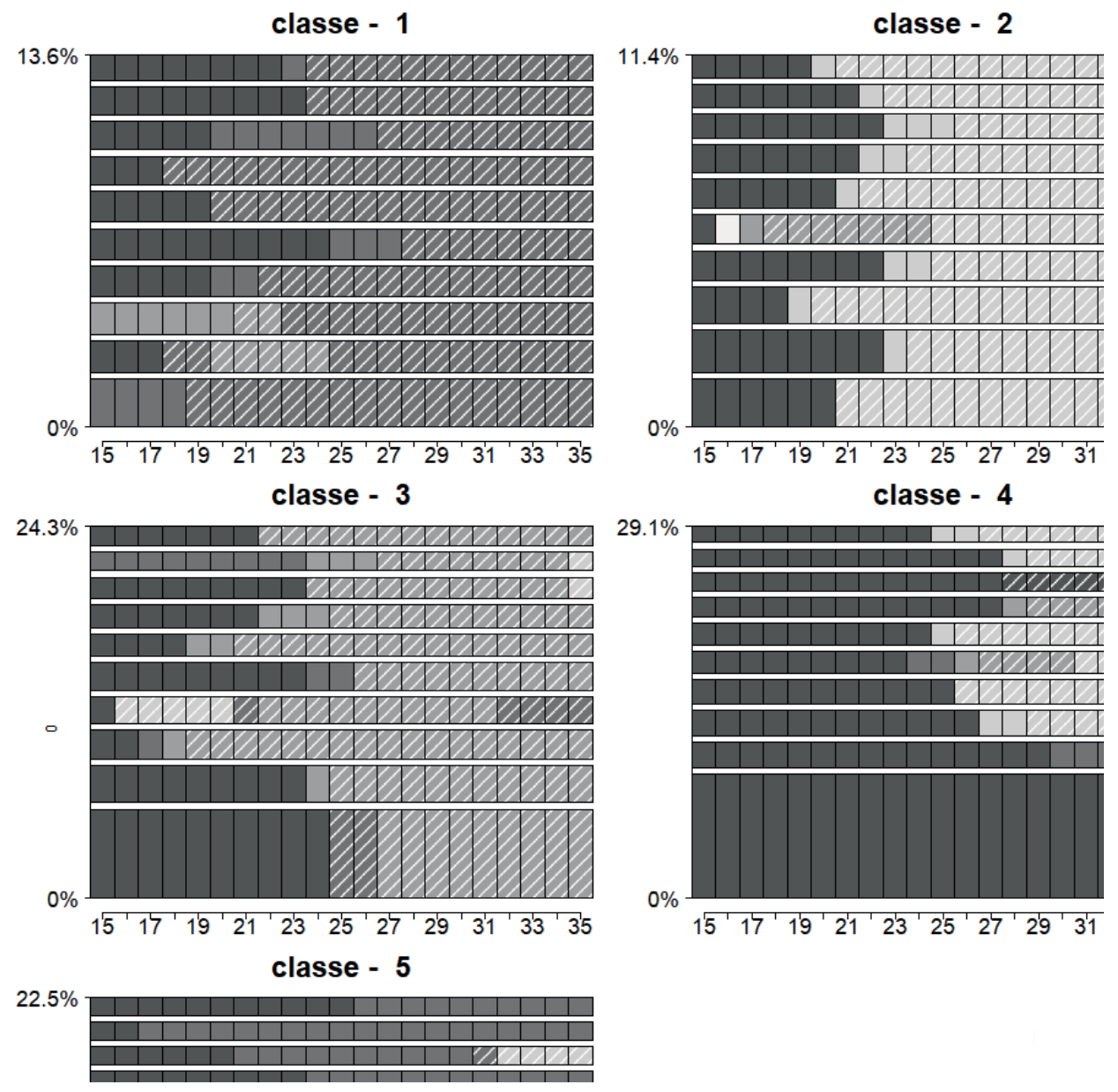

Figure 3 : Trajectoires familiales les plus fréquentes entre 15 et 35 ans Parmi chaque Classe - Femmes Source: MFV (INED, 2010) | Lecture : Les graphiques figurent les dix trajectoires les plus fréquentes dans chaque classe, en indiquant quel pourcentage de la classe en fait l'expérience. Ainsi, la trajectoire fréquente dans la classe 2 est quitter le domicile parental à 20 ans, au moment où surviennent ensemble le mariage et la naissance du premier enfant. Dans la classe 4, c'est vivre chez ses parents sans époux ni enfants de ses 15 à ses 35 ans. 

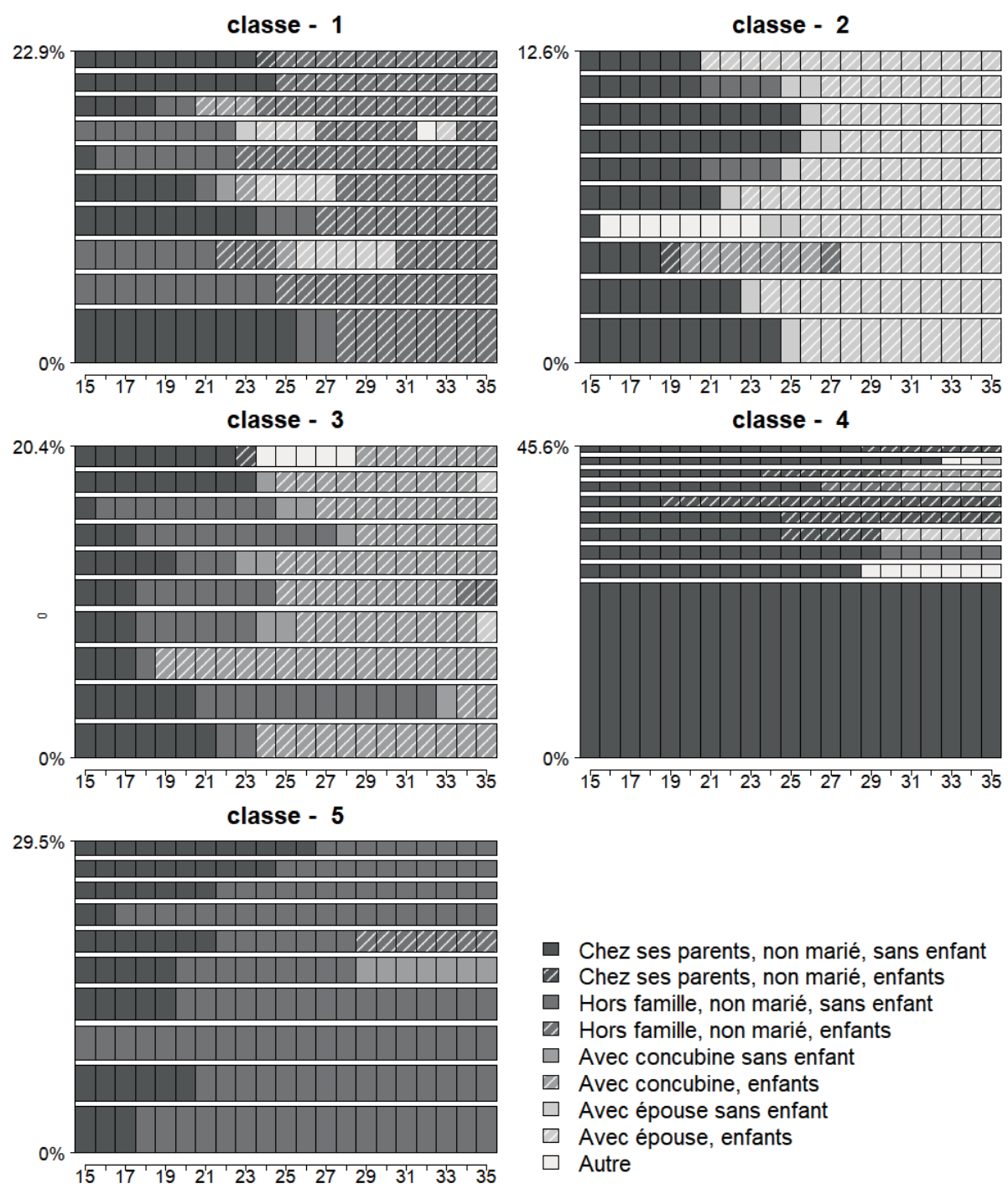

Figure 4 : Trajectoires familiales les plus fréquentes entre 15 et 35 ans parmi Chaque Classe - Hommes Source : MFV (INED, 2010)

Ces trajectoires reflètent les expériences répandues plus largement au sein des populations ultramarines, y compris non migrantes (Marie, Breton, 2015), mais font aussi ressortir le poids des formations familiales tardives (classes 4 et 5) pour les migrant.e.s de retour. Ces parcours génèrent des opportunités et des contraintes spécifiques, de telle sorte que famille et migration de retour s'influencent réciproquement : il est notamment fréquent que des femmes reviennent dans les DOM pour y élever leurs enfants né·e·s en métropole ou qu'une séparation survienne au moment où un des partenaires seulement retourne dans les DOM, union ou naissance d'enfants peuvent aussi être retardées par le séjour en métropole (Condon, Beaugendre, 2018). Pour saisir ces liens en tenant compte d'autres aspects des parcours, on étudie les caractéristiques 
des retours selon la trajectoire, puis on mesure l'effet du retour sur les événements familiaux et leur temporalité à l'aide de régressions.

\section{Des liens différenciés avec les conditions de retour}

Sur les figures 1 et 2, la période autour des 25 ans constitue un moment pivot, au cours duquel une grande partie des mises en union et naissances de premiers enfants ont lieu (plutôt un peu avant pour les femmes, un peu après pour les hommes). Pour les classes 1 à 3, elles interviennent peu après avoir quitté le domicile parental. Pour la classe 4, le départ du domicile parental intervient plus tard, voire pas avant 35 ans (les personnes ayant migré durant l'enfance n'y sont pas plus nombreuses). Pour la classe 5, le départ du domicile parental est en moyenne plus précoce (avant 20 ans) et non suivi d'une mise en couple. Pour comprendre comment ces trajectoires sont liées au retour, on commence donc par étudier comment la temporalité des retours varie selon le sexe et la trajectoire familiale ${ }^{8}$ (tableau 3).

Tableau 3 : Distribution des âges au retour du dernier séjour migratoire au sein des classes (\%)

\begin{tabular}{|l|c|c|c|c|c|c|}
\cline { 2 - 7 } \multicolumn{1}{c|}{} & \multicolumn{3}{c|}{ Femmes } & \multicolumn{3}{c|}{ Hommes } \\
\cline { 2 - 7 } \multicolumn{1}{l|}{} & $0-24$ & $25-34$ & $35^{+}$ & $0-24$ & $25-34$ & $35^{+}$ \\
\hline $\begin{array}{l}\text { Parentalité } \\
\text { hors union } \\
\text { cohabitante }\end{array}$ & 26,8 & 30,7 & 42,4 & 50,3 & 24,9 & 24,8 \\
\hline $\begin{array}{l}\text { Parentalité au } \\
\text { sein du } \\
\text { mariage }\end{array}$ & 23,7 & 40,2 & 36,0 & 47,9 & 20,4 & 31,7 \\
\hline $\begin{array}{l}\text { Parentalité au } \\
\text { sein du } \\
\text { concubinage }\end{array}$ & 24,7 & 34,8 & 40,6 & 56,5 & 22,9 & 20,5 \\
\hline $\begin{array}{l}\text { Départs tardifs } \\
\text { du domicile } \\
\text { parental }\end{array}$ & 18,3 & 37,0 & 44,7 & 50,4 & 25,6 & 24,0 \\
\hline $\begin{array}{l}\text { Décohabitation } \\
\text { sans couple ni } \\
\text { enfant }\end{array}$ & 21,5 & 28,2 & 50,4 & 44,9 & 22,4 & 32,7 \\
\hline Ensemble & 23,0 & 34,9 & 42,1 & 52,2 & 23,4 & 24,5 \\
\hline
\end{tabular}

Source : MFV (INED, 2010) | Champ : personnes âgées d'au moins 36 ans, nées dans les DOM et ayant vécu au moins six mois hors de leur DOM de naissance ${ }^{8}$ Cette distribution est soumise à un effet de troncature dans la mesure où les migrant·e·s de re-
tour les plus jeunes ont bien moins de chance d'avoir connu un retour après 35 ans. 
D'abord, les hommes reviennent dans les DOM plus tôt que les femmes : seul un quart des migrants de retour sont rentrés à 35 ans ou plus et plus de la moitié sont rentrés avant 25 ans ; parmi les migrantes de retour, au contraire, moins d'un quart sont rentrées avant 25 ans et plus de $40 \%$ à 35 ans ou plus. Cela s'explique en partie par le service militaire en métropole, qui a concerné la majorité des cohortes d'hommes étudiés (et pas les femmes), ce dernier étant un moteur de migrations courtes autour de 20 ans. L'association entre âge au retour et trajectoire familiale varie également selon le sexe. Les femmes qui connaissent une trajectoire de décohabitation sans couple ni enfant sont celles qui retournent les plus âgées (la moitié ne sont rentrées qu’à 35 ans ou plus), suivies par les femmes quittant tardivement le domicile parental (45\%). Les femmes dans les trajectoires de parentalité au sein du mariage sont celles qui rentrent les plus jeunes. Ainsi, on observe un gradient lié à l'union et à la parentalité, allant de la classe d'entrée dans le mariage avec enfants à celle du départ rapide du domicile parental sans union ni enfant, suggérant que plus les contraintes familiales sont fortes, plus le retour est précoce. Cette dynamique ne s'applique pas aux hommes. En effet, si ceux qui connaissent une trajectoire de décohabitation sans couple ni enfant sont ceux qui rentrent le plus tard, ceux qui connaissent une trajectoire de parentalité au sein du mariage sont également nombreux à rentrer à 35 ans ou plus (respectivement 33 et $32 \%$ ). Les hommes connaissant des trajectoires de départs tardifs du domicile parental rentrent plus fréquemment à un âge jeune (50 \% avant 25 ans), tout comme les pères non cohabitants (50 \% aussi) et moins que les pères au sein du concubinage (56 \%). Ces éléments montrent que les calendriers migratoires sont structurés par des logiques familiales et de genre, même s'ils ne nous permettent pas d'en déceler les mécanismes avec précision. Ils rappellent également qu'on peut comprendre la trajectoire familiale plutôt comme une cause ou comme une conséquence des conditions de retour, ce qui pose un enjeu méthodologique.

Tableau 4 : Distribution des conditions de retour après le dernier séjour migratoire au sein des classes (\%)

\begin{tabular}{|l|c|c|c|c|}
\cline { 2 - 5 } \multicolumn{1}{c|}{} & \multicolumn{2}{c|}{ Femmes } & \multicolumn{2}{c|}{ Hommes } \\
\cline { 2 - 5 } \multicolumn{1}{c|}{} & $\begin{array}{c}\text { Retour } \\
\text { contraint }\end{array}$ & Retour difficile & $\begin{array}{c}\text { Retour } \\
\text { contraint }\end{array}$ & Retour difficile \\
\hline $\begin{array}{l}\text { Parentalité hors } \\
\text { union cohabi- } \\
\text { tante }\end{array}$ & 21,0 & 31,3 & 16,6 & 18,5 \\
\hline $\begin{array}{l}\text { Parentalité au } \\
\text { sein du mariage }\end{array}$ & 26,6 & 30,4 & 24,0 & 19,0 \\
\hline $\begin{array}{l}\text { Parentalité au } \\
\text { sein du } \\
\text { concubinage }\end{array}$ & 19,3 & 26,3 & 22,0 & 24,8 \\
\hline
\end{tabular}




\begin{tabular}{|l|c|c|c|c|}
\hline $\begin{array}{l}\text { Départs tardifs } \\
\text { du domicile } \\
\text { parental }\end{array}$ & 31,3 & 39,2 & 18,6 & 27,3 \\
\hline $\begin{array}{l}\text { Décohabitation } \\
\text { sans couple ni } \\
\text { enfant }\end{array}$ & 21,7 & 32,1 & 16,8 & 27,1 \\
\hline Ensemble & 24,3 & 31,3 & 20,0 & 24,1 \\
\hline
\end{tabular}

Source : MFV (INED, 2010) | Champ : personnes âgées d'au moins 36 ans, nées dans les DOM et ayant vécu au moins six mois hors de leur DOM de naissance

Le tableau 4 reporte la distribution des conditions de retour selon les classes de trajectoires. Comme observé précédemment (tableau 1), les femmes connaissent plus souvent des difficultés au retour. Néanmoins, il existe d'importantes disparités selon le type de trajectoire et les groupes connaissant davantage de retours contraints ne sont pas toujours ceux rencontrant davantage de difficultés aux retours. Les femmes connaissant des départs tardifs sont celles qui expriment le plus souvent être rentrées dans les DOM par contrainte et avoir alors rencontré des difficultés (respectivement 31 et $39 \%$ ), ce qui suggère que la cohabitation prolongée avec les parents reflète des difficultés d'insertion pour ces femmes. Les femmes dans des trajectoires de parentalité au sein du mariage déclarent également plus fréquemment un retour contraint (27\%) et des difficultés (30\%), alors que c'est moins fréquent pour celles de la classe du concubinage (respectivement 19 et $26 \%$ ). Pour les hommes, les départs tardifs connaissent également plus de difficultés que les autres (27 \%) - autant que ceux dans des trajectoires de décohabitation sans couple ni enfant - mais pas plus de retours contraints. En revanche, les différences entre mariage et concubinage s'inversent : la déclaration de difficultés au retour est moins fréquente dans la classe du mariage et plus fréquente dans celle du concubinage (19 et $25 \%$ respectivement). Différentes configurations familiales apparaissent donc comme des vecteurs potentiels de contraintes et/ou de difficultés selon des modalités qui varient selon le sexe. Néanmoins, l'interprétation de ces écarts est limitée par notre capacité à situer ces retours contraints ou difficiles dans les calendriers des trajectoires.

Pour traiter ses différences, il faut distinguer les retours survenus avant la fin de la période d'observation de la trajectoire familiale de ceux survenus après. On se focalise ici sur les retours survenus avant 35 ans, sans exclure les personnes revenues après 35 ans. On applique des modèles de régression aux données longitudinales, en observant chaque personne aux mêmes âges (de 15 à 35 ans), quel que soit son âge au moment de l'enquête. Ne disposant d'information sur les conditions du retour que pour le dernier séjour migratoire, on se focalise sur les personnes n'ayant connu qu'une migration. On conserve 3110 personnes des 4015 de l'échantillon initial. On utilise des modèles de durée à temps discret pour étudier l'impact du retour sur les étapes des trajectoires : départ du domicile parental, arrivée du premier enfant, première mise en union cohabitante et premier mariage. Il s'agit de modèles de logistique avec pour 
variable expliquée l'indicatrice d'un événement qui ne peut arriver qu'une seule fois. On observe les individus chaque année à partir de 15 ans tant que l'événement n'est pas arrivé (la variable expliquée vaut alors 0). L’année où l'événement survient, la variable prend la valeur 1. Les années suivantes, l'individu quitte l'échantillon. Si l'événement ne survient pas avant 35 ans, on observe l'individu jusqu'à ses 35 ans (tableau 5). Comme on arrête les observations à 35 ans, certaines personnes n'ont pas connu leur retour vers les DOM avant la fin de la période d'observation, mais l'ensemble des personnes ayant connu une seule migration est inclus dans l'échantillon.

Tableau 5 : Structure des données pour le modèle logistique à temps discret expliquant la survenue de la première union cohabitante - valeurs prises par la variable « union cohabitante » au cours du temps pour trois individus fictifs

\begin{tabular}{|c|c|c|c|c|c|c|c|c|c|c|c|c|c|c|c|c|c|c|c|c|}
\hline 15 & 16 & 17 & 18 & 19 & 20 & 21 & 22 & 23 & 24 & 25 & 26 & 27 & 28 & 29 & 30 & 31 & 32 & 33 & 34 & 35 \\
\hline 0 & 0 & 0 & 0 & 0 & 0 & 0 & 0 & 0 & 0 & 0 & 0 & 0 & 0 & 0 & 0 & 0 & 0 & 0 & 0 & 0 \\
\hline 0 & 0 & 0 & 0 & 0 & 0 & 0 & 0 & 0 & 0 & 0 & 0 & 1 &. &. &. &. &. &. &. &. \\
\hline 0 & 0 & 0 & 0 & 0 & 0 & 1 &. &. &. &. &. &. &. &. &. &. &. &. &. &. \\
\hline
\end{tabular}

Lecture : la première personne observée n'a pas connu d'union cohabitante entre ses 15 et 35 ans, la deuxième a commencé à cohabiter avec un·e conjoint·e à 27 ans, la troisième à 21 ans.

Les modèles sont spécifiés comme suit:

$$
\log \left(\frac{\mathbb{P}\left(Y_{i t}=1\right)}{\mathbb{P}\left(Y_{i t}=0\right)}\right)=\beta_{0}+\beta_{1} X_{i t}+\varepsilon_{i t}
$$

Où $\mathrm{Y}_{\mathrm{it}}$ est la valeur prise par l'indicatrice de l'événement expliqué pour l'individu i à l'âge $\mathrm{t}(\mathrm{t} \in \llbracket 15 ; 35 \rrbracket)$, $\mathrm{X}_{\mathrm{it}}$ un vecteur de caractéristiques individuelles qui peuvent varier dans le temps, $\beta_{1}$ le vecteur de coefficients estimés par le modèle, $\beta_{0}$ la constante et $\varepsilon_{\text {it }}$ le terme d'erreur.

Ces modèles permettent d'étudier finement la temporalité des événements, en tenant compte de l'ordre dans lequel ces derniers arrivent. On capture les chances qu'une étape survienne ou non, mais aussi la vitesse à laquelle elle survient. Ils ont pour variables de contrôle : l'âge (une indicatrice pour chaque âge), le niveau d'études (encore étudiant, CEP ou aucun diplôme, Brevet, CAP ou BEP, Bac'), le fait d'avoir connu ou non son premier emploi, la cohorte de naissance (1929-1945, 1946-1955, 1956-1965, 1966-1975), le DOM de naissance, l'expérience migratoire et son interaction avec le sexe. Seuls le sexe, la cohorte et le DOM ne peuvent pas varier au cours du temps pour une même personne. Deux variables catégorielles mesurent l'expérience migratoire et sont utilisées dans des modèles séparés. La première compare les statuts suivants : n’a jamais quitté le DOM ; est en migration; est revenu dans les DOM par contrainte ; est revenu dans les DOM par choix. La deuxième compare les statuts suivants : n'a jamais quitté le DOM ; est en migration ; est revenu dans les DOM dans des conditions difficiles ; est revenu

\footnotetext{
${ }^{9}$ Les effectifs de personnes ayant achevé des études supérieures dans ces cohortes de 35 ans et plus sont trop faibles pour que distinguer les personnes ayant le baccalauréat de celles ayant un diplôme de l'enseignement supérieur conduise à des analyses robustes.
} 
dans les DOM sans difficulté. Les résultats complets sont consignés dans les tableaux A1 et $\mathrm{A} 2$ en annexe. Les figures 3 à 6 présentent les effets de l'expérience migratoire selon le sexe exprimés sous des effets marginaux (les deux variables d'expérience migratoire sont présentées dans la même figure). La modalité de référence est : « en migration ».

Rentrer dans les DOM plutôt qu'être en migration diminue les chances de quitter le domicile parental. Cet effet négatif est significativement plus fort pour les femmes que pour les hommes. On n'observe pas de différence selon les conditions du retour. Bien sûr, cet effet concerne des personnes qui ont migré avec leur(s) parent(s) et n'ont pas quitté le domicile parental avant le retour, un groupe très spécifique de notre échantillon. Néanmoins, ces résultats offrent un éclairage pour mieux comprendre les trajectoires de départ tardif identifiées dans la partie précédente. Pour les hommes, le retour augmente les chances d'avoir un premier enfant, quand il s'effectue sans contrainte ou sans difficulté. En revanche, on n'observe pas un tel effet pour les femmes, voire un effet négatif quand le retour est associé à des difficultés de réinstallation. Cette dynamique est plus marquée quand on considère les unions. Pour les unions cohabitantes comme pour les mariages, le retour diminue les chances d'union pour les femmes (cet effet apparaît plus fort quand le retour est associé à une contrainte ou des difficultés, même si la différence n'est pas significative) et les augmente légèrement pour les hommes ou n'a pas d'effet significatif. 


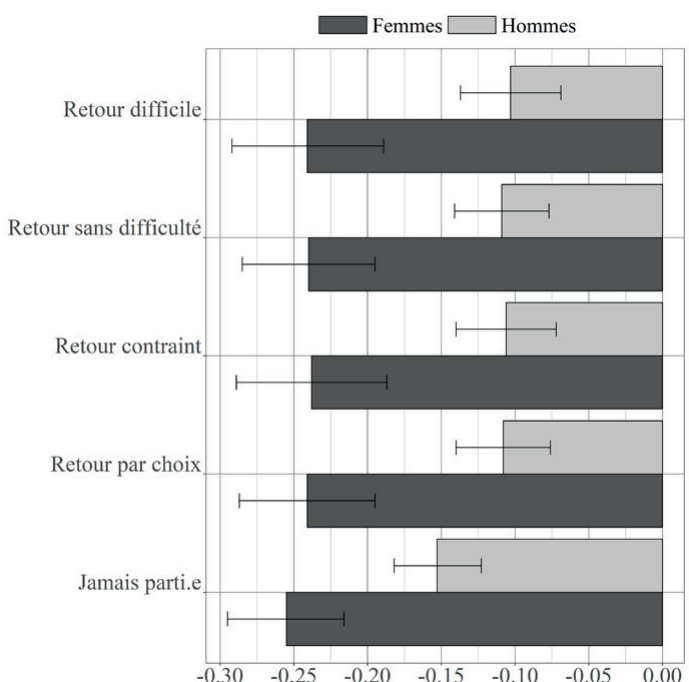

FIGURE 5 : EfFET MARGINAL DE L'EXPÉRIENCE MIGRATOIRE SUR LES CHANCES DE QUITTER LE DOMICILE PARENTAL

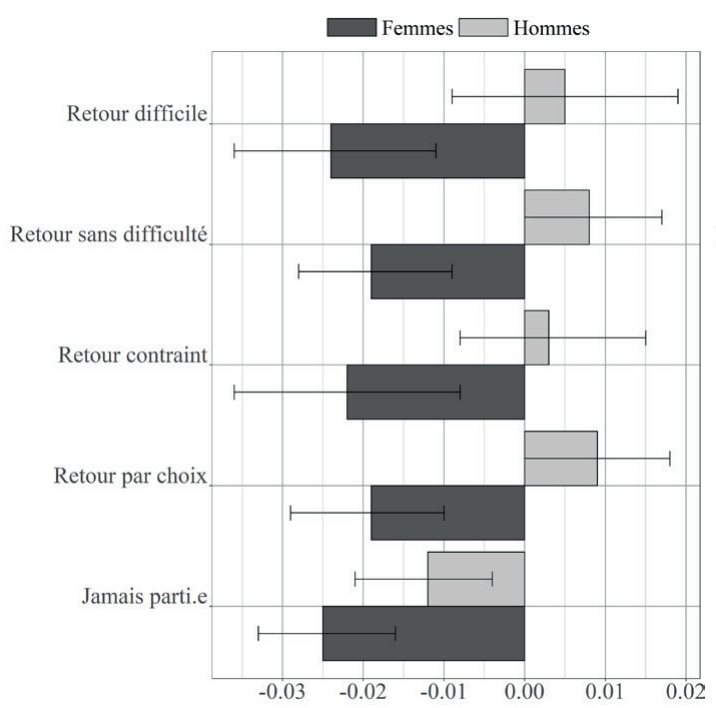

FIGURE 7 : EfFET MARGINAL DE L'EXPÉRIENCE MIGRATOIRE SUR LES CHANCES DE PREMIÈRE UNION COHABITANTE

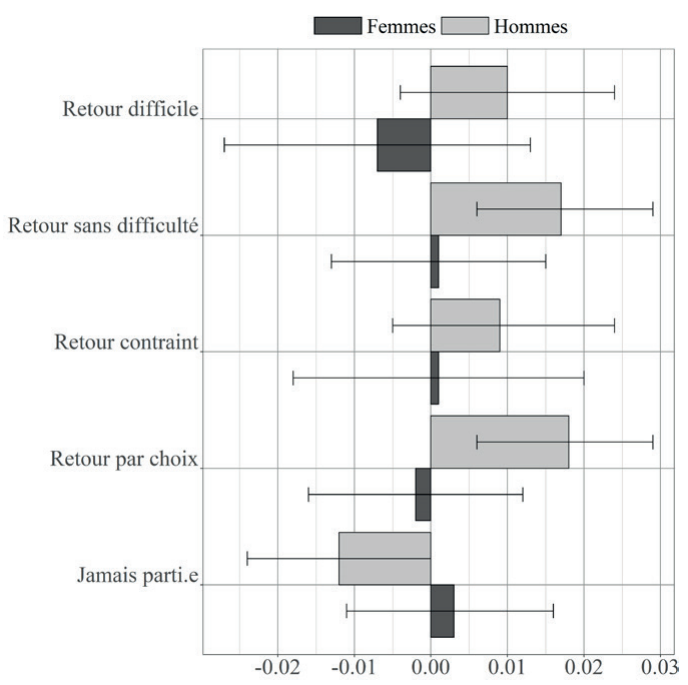

FIGURE 6 : EFFET MARGINAL DE L'EXPÉRIENCE MIGRATOIRE SUR LES CHANCES D'AVOIR SON PREMIER ENFANT

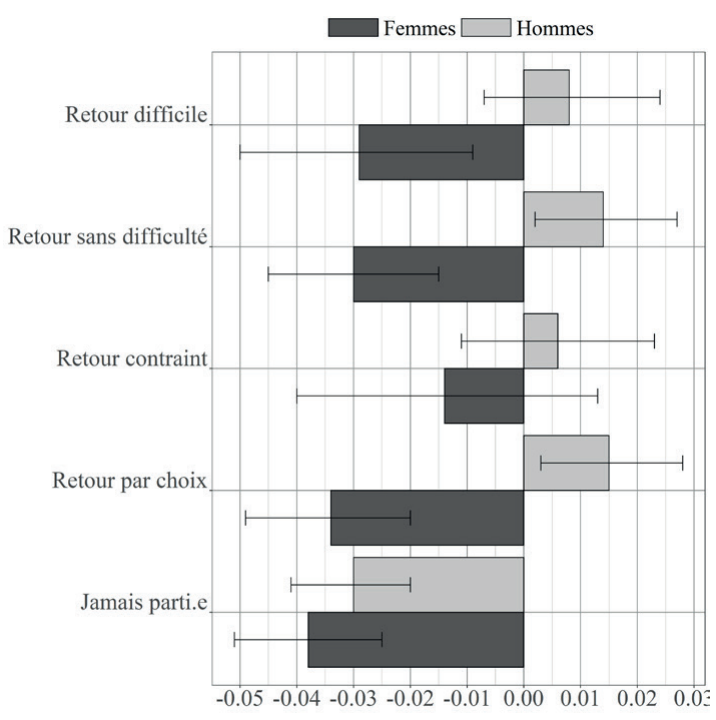

FIGURE 8 : EfFET MARGINAL DE L'EXPÉRIENCE MIGRATOIRE SUR LES CHANCES DE PREMIER MARIAGE

Source : MFV (INED, 2010) | Lecture : pour les femmes, vivre dans les DOM après un retour difficile plutôt qu'être en migration diminue de 23 points de probabilité les chances de quitter le domicile parental une année donnée.

L'impact genré du retour et des conditions dans lesquelles il s'effectue sur les unions apparaît encore plus marqué lorsque l'on considère les transitions conjugales : la constitution et la rupture des unions (cohabitation, mariage). Des modèles de panel à effets fixes mesurent l'effet de caractéristiques qui varient dans le temps, net de l'effet des caractéristiques fixes des individus, y compris celles que l'enquête ne permet pas 
d'observer. La mesure de cet effet net est permise par l'estimation d'une constante par individu, au lieu d'une constante pour l'ensemble du modèle, ce qui est rendu possible car on observe les mêmes personnes plusieurs fois dans le temps. Dans ces modèles, on observe les individus de leurs 15 à leurs 35 ans, mais seuls les individus qui connaissent au moins un changement dans la variable expliquée sont pris en compte (1 442 et 898 individus respectivement pour la cohabitation et le mariage). Ainsi, on estime les chances de passer d'un état à un autre. Cette fois-ci, la notion de durée n'est pas prise en compte (on observe tous les individus à tous les âges). Les modèles ont pour variables de contrôle : l'âge, le fait d'avoir ou non fini ses études, le fait d'avoir connu ou non son premier emploi, l'expérience migratoire et son interaction avec le sexe.

Les modèles sont spécifiés comme suit:

$$
\log \left(\frac{\mathbb{P}\left(Y_{i t}=1\right)}{\mathbb{P}\left(Y_{i t}=0\right)}\right)=\beta_{0}+\beta_{1} X_{i t}+\varepsilon_{i t}
$$

Où $\mathrm{Y}_{\text {it }}$ est la valeur prise par l'indicatrice expliquée pour l'individu i à l'âge $t$ ( $t \in \llbracket 15$; 35】), $\mathrm{X}_{\mathrm{it}}$ un vecteur de caractéristiques individuelles qui peuvent varier dans le temps, $\beta_{1}$ le vecteur de coefficients estimés par le modèle, $\alpha_{\mathrm{i}}$ la constante pour l'individu i et $\varepsilon_{\mathrm{it}}$ le terme d'erreur.

Les figures 7 et 8 présentent les effets de l'expérience migratoire selon le sexe exprimés sous forme d'odds ratios ${ }^{10}$ et les résultats complets sont consignés dans le tableau A3 en annexe. Pour les hommes, quelles que soient les conditions du retour, rentrer dans les DOM augmente les chances d'être en union, même si cet effet positif est plus fort quand le retour s'effectue par choix ou sans difficulté. Pour les femmes, rentrer dans les DOM diminue les chances d'être en union, mais l'effet négatif est significativement plus important lorsque le retour est contraint. Lorsqu'on différencie le retour en fonction des difficultés rencontrées, un retour sans difficulté n'a pas d'effet significatif alors qu'un retour difficile diminue les chances d'être en union. Autrement dit, les hommes ont plus de chances de se marier ou de vivre en couple après leur retour que durant leur migration, alors que les femmes ont plus de chances de connaître une séparation après leur retour que durant leur migration.

\footnotetext{
${ }^{10}$ Les odds ratios mesurent le rapport entre les cotes, ou odds, d'un événement pour deux groupes d'observation. Contrairement à la probabilité, qui mesure « une migrante de retour sur deux est mariée » (la probabilité vaut 1/2), la cote mesure « pour une migrante de retour mariée, il y en a une autre qui ne l'est pas » (la cote vaut 1). Dans les modèles exposés ici, les odds ratios mesurent donc le ratio entre la cote d'être marié.e (ou en union cohabitante selon le modèle) pour la modalité observée et la cote d'être marié.e pour la modalité de référence. Par simplification, on interprète ces ratios comme des rapports de chances.
} 


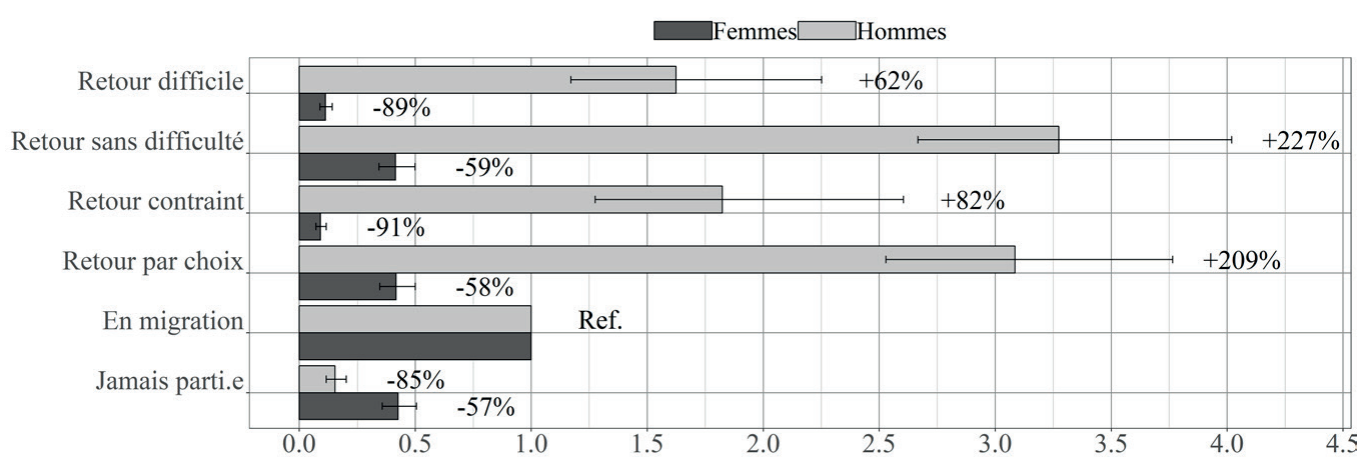

FIGURE 9 : EFFET DE L'EXPÉRIENCE MIGRATOIRE SUR L'UNION COHABITANTE EXPRIMÉ SOUS FORME D'ODDS RATIOS Source : MFV (INED, 2010)| Lecture : connaître un retour difficile plutôt que de rester en migration augmente les chances de se mettre en couple cohabitant de $62 \%$ pour les hommes et les diminue de $89 \%$ pour les femmes.

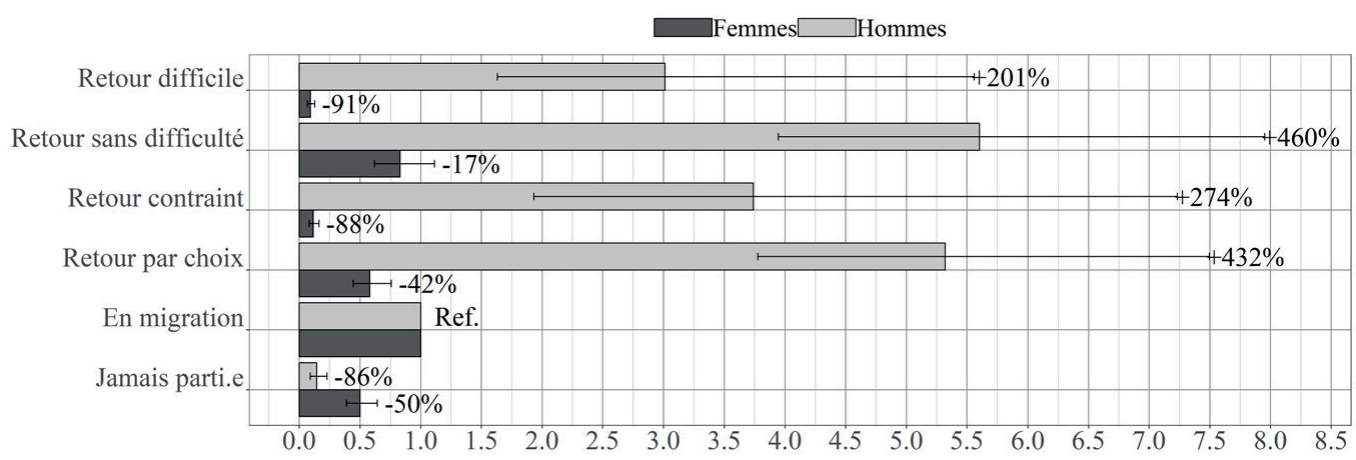

FIGURE 10 : EFFET DE L'EXPÉRIENCE MIGRATOIRE SUR LE MARIAGE EXPRIMÉ SOUS FORME D'ODDS RATIOS Lecture : connaître un retour difficile plutôt que de rester en migration augmente les chances de se marier de $201 \%$ pour les hommes et les diminue de $91 \%$ pour les femmes.

Ces résultats offrent plusieurs pistes d'interprétation. D’abord, ils nous renseignent sur la manière dont les calendriers des histoires de vie sont organisés ou réorganisés autour de la migration. Les séjours migratoires des femmes durent en moyenne plus longtemps que ceux des hommes : 5,6 ans pour les hommes contre 8,9 ans pour les femmes dans notre échantillon réduit. Les femmes connaissent également leur migration plus tard que les hommes : les hommes de cet échantillon quittent leur DOM de naissance en moyenne à 21,7 ans, les femmes à 25,5 ans. Enfin, les femmes se marient plus tôt que les hommes (24,7 ans contre 26,5) et font l'expérience de leur première union et premier enfant plus jeune. Ainsi, pour les hommes, la migration intervient avant la mise en couple et l'union survient après le retour. La migration est davantage vécue comme une parenthèse précédant la formation du couple et de la famille dans les DOM. Pour les femmes, la migration arrive plus souvent après la mise en couple ou peu de temps avant. La formation du couple et de la famille se fait principalement durant la migration, qui dure plus longtemps. Si elles n'ont pas lieu durant la migration, les naissances d'enfants ou unions se trouvent encore retardées par le retour. Si elles 
ont eu lieu avant le retour, ce dernier intervient comme un épisode perturbateur, favorisant la séparation, surtout lorsqu'il est vécu comme une contrainte ou associé à des difficultés de réinstallation. La famille apparaît donc pour les femmes étudiées comme un vecteur de contraintes, peu compatible avec l'autonomie liée à la circulation entre régions éloignées. D’autre part, on peut également interpréter ces résultats en termes de marchés matrimoniaux : les ultramarines de retour auraient eu plus de chances de trouver un.e partenaire en métropole que dans les DOM, alors que les ultramarins de retour utiliseraient l'expérience métropolitaine comme un capital pour augmenter leurs chances de trouver un·e partenaire une fois rentrés. Pour aller plus loin dans l'étude de ces questions, il serait nécessaire d'identifier le lieu de naissance des partenaires. Dans MFV, ce n'est malheureusement possible que pour les partenaires de couples encore unis au moment de l'enquête.

\section{Conclusion}

À l'aide d'analyses de séquence portant sur la formation de la famille et les modes de cohabitation, cet article met en valeur les trajectoires familiales typiques dont ont fait l'expérience les migrant.e.s de retour dans les DOM entre leurs 15 et 35 ans. Cinq types de trajectoires sont mis en valeur : l'entrée plutôt rapide dans la parentalité, en dehors d'une union, dans le cadre d'un couple cohabitant, ou dans le cadre d'un couple marié ; le départ tardif du foyer familial ; la décohabitation non suivie (ou tardivement) d'union et/ou de parentalité. Puis, des modèles de régression évaluent l'effet du retour avant 35 ans sur les étapes de formation de la famille (unions, enfants) selon le sexe, en distinguant les retours associés à des contraintes ou difficultés. Si elles tiennent compte des différences internes aux Outre-mer en contrôlant pour la région de naissance, ces estimations ne nous permettent pas d'éclairer les spécificités de chaque DOM. Mis à part le profil démographique similaire de la Guadeloupe et de la Martinique, les normes familiales et le poids de certaines configurations varient entre les DOM, par exemple du fait de l'importance de l'immigration en Guyane. L’approche développée ici se focalise sur leurs points communs : la forte prévalence de l'émigration, la réponse publique à des taux de fécondité élevés qui a orchestré la transition démographique des années 1960 et 1970, la variété des configurations familiales qui incluent plus souvent qu'en métropole des naissances d'enfants hors union (Beaugendre, Breton, Condon, 2018 ; Breton, 2011). Les analyses montrent alors que les calendriers migratoires et familiaux s'organisent selon des logiques de genre, avec un rapport différent à la contrainte. Quelles que soient ses conditions, le retour accélère la mise en couple et la naissance d'enfants pour les hommes. Pour les femmes, au contraire, rentrer dans les DOM retarde ces étapes et fragilise les unions si elles ont déjà eu lieu, d'autant plus si le retour est associé à des contraintes ou difficultés.

Ces résultats s'inscrivent dans un contexte spécifique. D’abord, ils portent par définition seulement sur les migrante.s qui ont connu un retour. Nos modèles considérant les trajectoires au cours de la vie, ils comparent le fait d'être encore dans les 
DOM, en migration ou de retour pour une même personne, mais ils ne traitent pas de la spécificité de son profil, comparé aux profils de celles et ceux qui ne migreront ou ne rentreront jamais. Ensuite, ils ne mesurent l'effet du retour et des conditions dans lesquelles il s'effectue que lorsque ce retour a lieu avant 35 ans, alors que les retours après cet âge sont fréquents. Cette restriction a du sens : on cherche à étudier le potentiel effet perturbateur des retours sur les trajectoires familiales et les grandes étapes de la vie familiale ont le plus souvent lieu avant 35 ans. Elle nous permet également de mesurer l'effet d'avoir connu un retour à ces âges, net de la sélection de la migration de retour en général, puisqu'on compare des personnes qui sont rentrées avant et après 35 ans. Enfin, nos résultats ne concernent que les cohortes migratoires les plus anciennes. La majorité de notre échantillon a migré avant 1983, pendant la période d'activité du Bureau pour la migration des DOM qui a marqué un moment particulier pour ces migrations (51\%), voire avant (7\%). Seuls $7 \%$ ont migré après l'abrogation du service militaire en 1997. Alors qu'aujourd'hui les migrations s'inscrivent souvent dans des parcours d'études ou de formation, celles que nous observons se segmentent principalement entre des mobilités liées au service militaire, à un recrutement ou une recherche d'emploi et à des logiques de rapprochement familial. Alors qu'ils relèvent de mécanismes distincts, ces trois canaux ne sont pas également distribués entre hommes et femmes (Condon, 2004 ; 2008), ce qui explique en partie les différences genrées observées. Néanmoins, nos analyses vont plus loin que l'identification de canaux de migration différenciés selon le sexe : elles en révèlent certains des mécanismes sousjacents et soulignent leurs liens étroits avec les trajectoires familiales. Ainsi, cette étude renforce les conclusions de précédents travaux portant sur les difficultés spécifiques rencontrées par les femmes au moment du retour dans les DOM (Condon, Beaugendre, 2018) et suggère que les circulations depuis les DOM sont associées à un besoin accru d'autonomie pour ces dernières, sur lesquelles les contraintes familiales pèsent plus fortement que sur les hommes. Elle contribue à une sociologie des migrations attentive à la sphère familiale et domestique, en mettant en valeur l'effet des modalités de retour, plus rarement étudiées que celles des départs. Elle invite enfin à étudier de manière plus approfondie les logiques de circulation entre DOM et métropole, sous l'angle des marchés matrimoniaux.

\section{Bibliographie}

Anselin A. (1990), L'émigration antillaise en France : la troisième île, Paris, Karthala.

AtTiAs-Donfut C., LAPIERRE N. (1997), La Famille providence : trois générations en Guadeloupe, Paris, La Documentation française.

Beaugendre C., Breton D., Condon S. (2018), « Faire couple aux Antilles : Contre vents et marées ? », in J.-P. SANDERSON, M. ORIs, Familles en transformation. Quand les modes de construction familiale se réinventent, Paris, AIDELF, p. 5-24. 
BRETON D. (2011), « L'entrée dans l'âge adulte des jeunes réunionnaises et martiniquaise : la famille comme une "évidence" ?», Revue des politiques sociales et familiales, vol.106, $\mathrm{n}^{\circ} 1$, p. 25-38.

Brinbaum Y., SAFi M., Simon P. (2012), Les discriminations en France : entre perception et expérience, Documents de travail, $\mathrm{n}^{\circ} 183$, INED.

Condon S. (1996), «Les Migrants antillais en métropole : un espace de vie transatlantique», Espace, populations, sociétés, vol. 14, n² 2, p. 513-520.

Condon S. (2004), « Gender Issues in the Study of Circulation between the Caribbean and the French Metropole », Caribbean Studies, vol. 32, n 1, p. 129-159.

Condon S. (2008), « Travail et genre dans l'histoire des migrations antillaises », Travail, genre et sociétés, vol. 20, p. 67-86.

Condon S., Beaugendre C. (2018), « Partir pour tourner la page : migrations entre les départements d'outremer français et l'Hexagone après une rupture conjugale », in S. Pennec, C. Girard, J.-P. Sanderson, Trajectoires et âges de la vie. Actes du colloque de l'Association internationale des démographes de langue française, Paris, AIDELF. En ligne. URL :https://www.erudit.org/en/books/actes-des-colloques-de-lassociationinternationale-des-demographes-de-langue-francaise/trajectoires-ages-vieselection-darticles-issus-travaux-presentes-au-xviiie--978-2-9521220-5-4/000444li/.

Condon S., Byron M. (2007), Migration in Comparative Perspective: Caribbean Communities in Britain and France, New York, Routledge.

Condon S., Ogden P.E. (1991), « Emigration from the French Caribbean: the Origins of an Organized Migration », International Journal of Urban and Regional Research, vol. 15, $\mathrm{n}^{\circ}$ 4, p. 505-523.

Constant F. (1987), « La politique française de l'immigration antillaise de 1946 à 1987 », Revue européenne de migrations internationales, vol. 3, nº 3, p. 9-30.

De Coulon A., Piracha M. (2005), « Self-selection and the performance of return migrants: the source country perspective », Journal of Population Economics, vol. 18, n 4 , p. 779807.

Domenach H., Picouet M. (1992), La Dimension migratoire des Antilles, Paris, Economica.

Haddad M. (2018), « Des minorités pas comme les autres ? Le vécu des discriminations et du racisme des ultramarins en métropole ", Revue française de sociologie, vol. 59, $\mathrm{n}^{\circ}$ 4, p. 649-676.

Hondagneu-Sotelo P., Avila E. (1997), « "I'm here, but I'm there" the meanings of Latina transnational motherhood », Gender e Society, vol. 11, n 5, p. 548-571.

Hugo G.J. (1981), "Village-Community Ties, Village Norms, and Ethnic and Social Networks: A Review of Evidence from the Third World », in G.F.D. JONG, R.W. GARDNER (dir.), Migration Decision Making, Oxford, Pergamon, p. 186-224. 
IHADDADENE F. (2017), « Les pratiques des professionnels face aux freins à la mobilité des jeunes ultramarins : l'exemple de La Réunion », Cahiers de l'action, vol. 49, p. 39-46.

MacIndoe H., Аввотт A. (2004), « Sequence Analysis and Optimal Matching Techniques for Social Science Data », in M.A. Hardy, A. Bryman (dir.), Handbook of Data Analysis, Londres, Sage, p. 387-406.

Marie C.-V., Breton D. (2015), " Les "modèles familiaux" dans les DOM : entre bouleversements et permanence. Ce que nous apprend l'enquête Migrations, famille et vieillissement ", Revue des politiques sociales et familiales, vol. 119, p. 55-64.

Marie C.-V., Rallu J.-L. (2004), « Migrations croisées entre DOM et Métropole : l'emploi comme moteur de la migration », Espace populations sociétés, vol. 1, n 2, p. 237-252.

Massey D.S., Alarcon R. el, Durand J., González H. (1990), Return to Aztlan: The Social Process of International Migration from Western Mexico, Berkeley, University of California Press.

MelÉndez E. (2017), Sponsored Migration: The State and Puerto Rican Postwar Migration to the United States, Colombus, Ohio State University Press.

Olwig K.F. (2007), Caribbean Journeys: An Ethnography of Migration and Home in Three Family Networks, Durham, Duke University Press Books.

Potter R.B., Conway D., Phillips J. (dir.) (2005), The experience of return migration: Caribbean perspectives, Aldershot, Ashgate.

Robette N., Thibault N. (2008), " Analyse harmonique qualitative ou méthodes d'appariement optimal ? Une analyse exploratoire de trajectoires professionnelles », Population (French Edition), vol. 63, nº 4, p. 621-646.

SKELDON R. (2012), « Going round in circles: Circular migration, poverty alleviation and marginality », International Migration, vol. 50, $n^{\circ}$ 3, p. 43-60.

Temporal F., Marie C.-V., Bernard S. (2011), « Insertion professionnelle des jeunes ultramarins : DOM ou métropole ?», Population, vol. 66, n 3, p. 555-599. 


\section{Annexes}
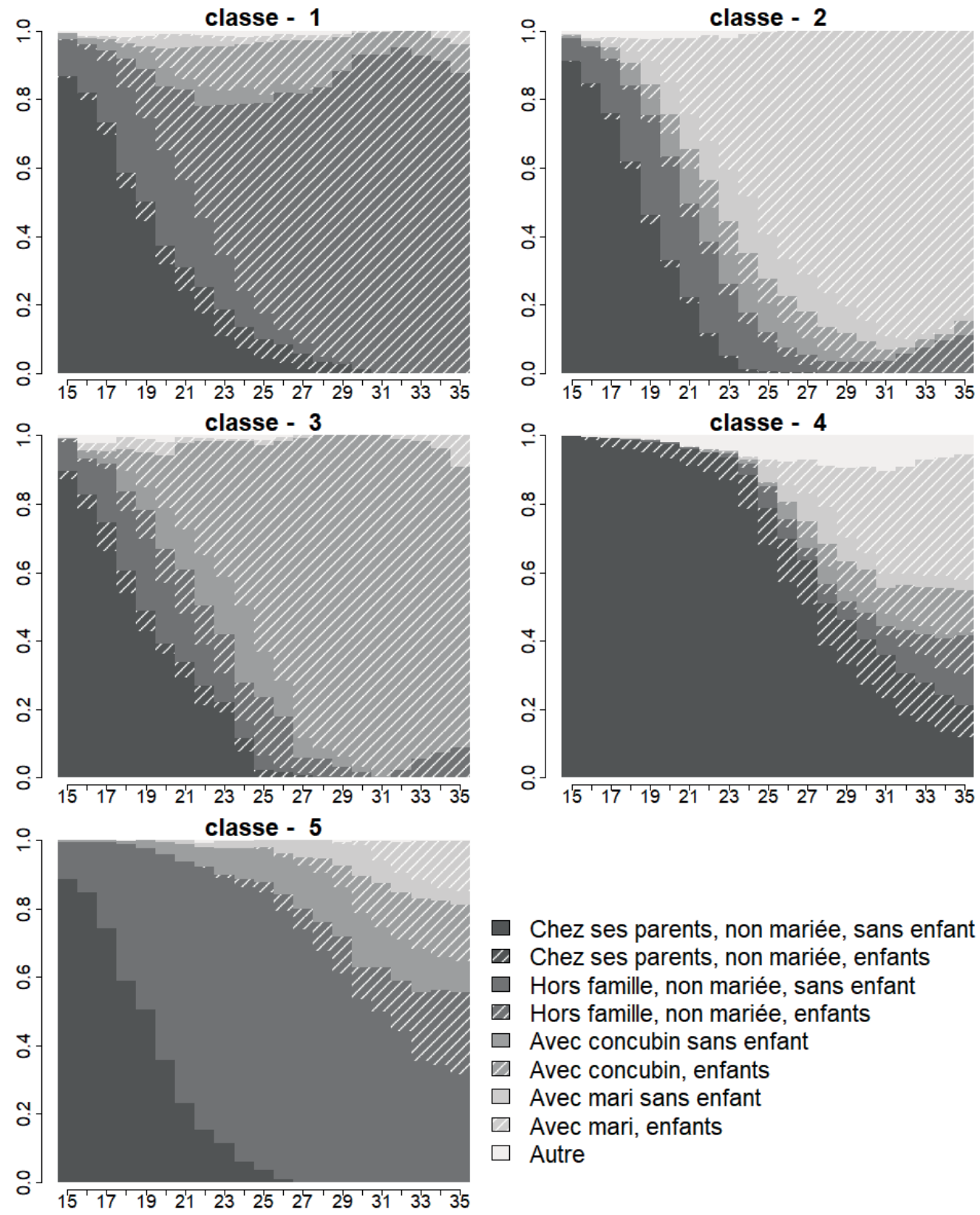

Chez ses parents, non mariée, sans enfant

$\checkmark$ Chez ses parents, non mariée, enfants

$\square$ Hors famille, non mariée, sans enfant

$\square$ Hors famille, non mariée, enfants

$\square$ Avec concubin sans enfant

$\square$ Avec concubin, enfants

$\square$ Avec mari sans enfant

$\square$ Avec mari, enfants

$\square$ Autre

Figure A1 : Distribution des CONFIgURATIONS FAMILIALES DANS CHAQUE CLASSE AU FIL DES ÂGES DES FEMMES Source : MFV (INED, 2010) 

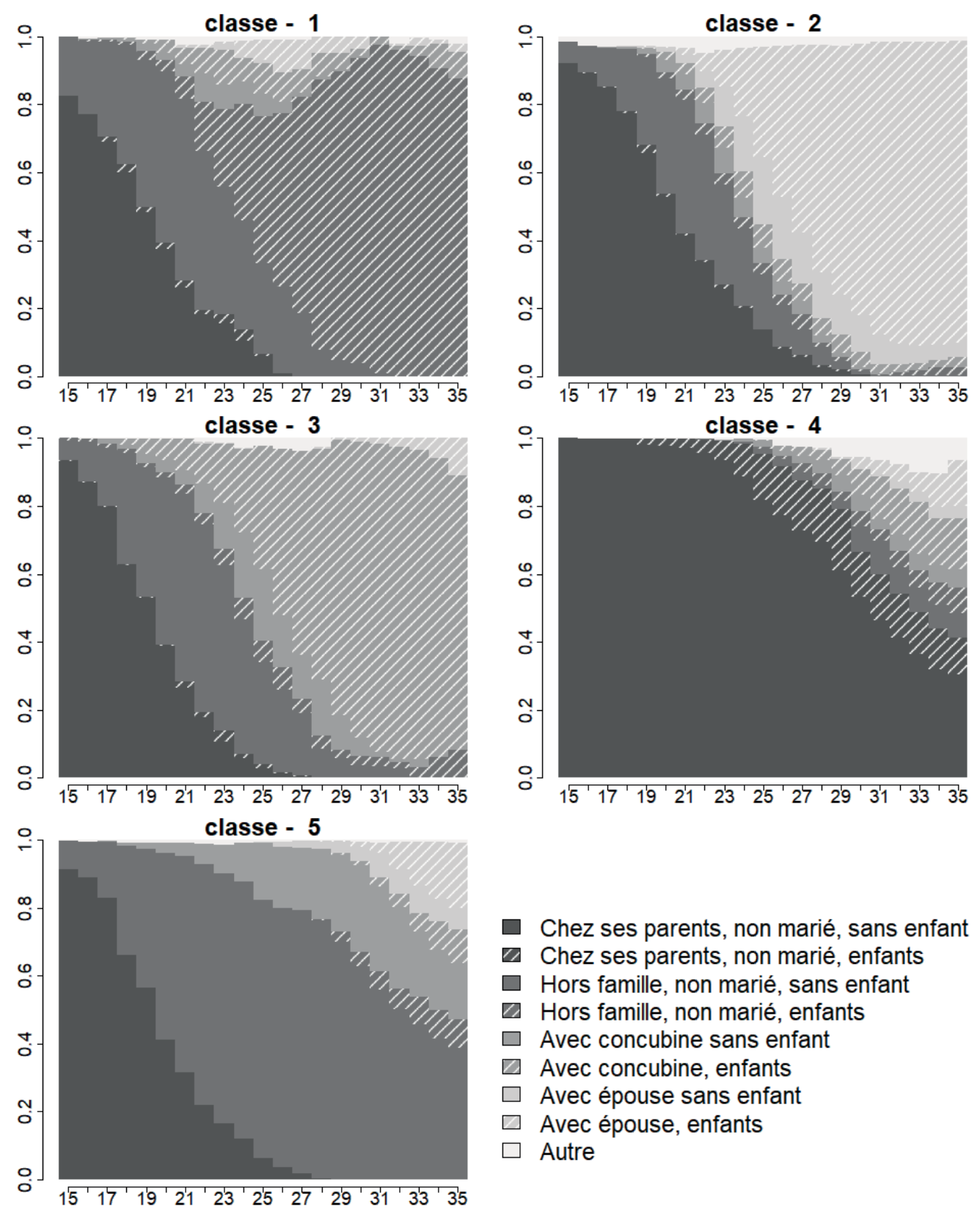

Chez ses parents, non marié, sans enfant

$\square$ Chez ses parents, non marié, enfants

$\square$ Hors famille, non marié, sans enfant

$\square$ Hors famille, non marié, enfants

$\square$ Avec concubine sans enfant

$\square$ Avec concubine, enfants

$\square$ Avec épouse sans enfant

$\square$ Avec épouse, enfants

$\square$ Autre

Figure A2 : Distribution des CONFIGURATIONS FAMILIALES DANS CHAQUE CLASSE AU FIL DES ÂGES DES HOMMES Source : MFV (INED, 2010) 
Tableau A1 : Résultats des modèles de durée exprimés sous forme d'odds ratios (difficulté)

\begin{tabular}{|c|c|c|c|c|c|c|c|c|}
\hline & \multicolumn{2}{|c|}{$\begin{array}{l}\text { Départ domicile } \\
\text { parental }\end{array}$} & \multicolumn{2}{|c|}{ Premier enfant } & \multicolumn{2}{|c|}{$\begin{array}{l}\text { Première union } \\
\text { cohabitante }\end{array}$} & \multicolumn{2}{|c|}{ Premier mariage } \\
\hline \multicolumn{9}{|c|}{ Âge de 15 (ref.) à 35 ans : coefficients omis } \\
\hline $\begin{array}{l}\text { Bac ou } \\
\text { plus }\end{array}$ & $1.56^{* * *}$ & {$[1.28,1.90]$} & $0.84^{* *}$ & {$[0.72,0.97]$} & $1.36^{* * *}$ & {$[1.17,1.58]$} & $1.85^{* * *}$ & {$[1.56,2.18]$} \\
\hline Brevet & $1.43^{* * *}$ & {$[1.18,1.74]$} & 0.93 & {$[0.78,1.12]$} & $1.27^{* *}$ & {$[1.05,1.53]$} & $1.57^{* * *}$ & {$[1.25,1.98]$} \\
\hline CAP, BEP & $1.16^{*}$ & {$[0.99,1.37]$} & 0.91 & {$[0.79,1.05]$} & 1.04 & {$[0.89,1.21]$} & 1.06 & {$[0.87,1.29]$} \\
\hline $\begin{array}{l}\text { CEP, } \\
\text { aucun }\end{array}$ & 1.00 & {$[1.00,1.00]$} & 1.00 & {$[1.00,1.00]$} & 1.00 & {$[1.00,1.00]$} & 1.00 & {$[1.00,1.00]$} \\
\hline Étudiant & 0.88 & {$[0.72,1.08]$} & $0.34^{* * *}$ & {$[0.25,0.45]$} & $0.57^{* * *}$ & {$[0.41,0.79]$} & 0.79 & {$[0.52,1.19]$} \\
\hline $1^{\text {er }}$ emploi & $1.49^{* * *}$ & {$[1.26,1.76]$} & $1.32^{* * *}$ & {$[1.11,1.57]$} & $1.49^{* * *}$ & {$[1.22,1.82]$} & $1.56^{* * *}$ & {$[1.24,1.96]$} \\
\hline $\begin{array}{l}\text { Guade- } \\
\text { loupe }\end{array}$ & Ref. & & Ref. & & Ref. & & Ref. & \\
\hline $\begin{array}{l}\text { Marti- } \\
\text { nique }\end{array}$ & 1.02 & {$[0.89,1.16]$} & 1.01 & {$[0.90,1.14]$} & 1.03 & {$[0.91,1.16]$} & 1.02 & {$[0.88,1.17]$} \\
\hline Guyane & $1.44^{* *}$ & {$[1.05,1.98]$} & $1.55^{* * *}$ & {$[1.25,1.93]$} & 1.19 & {$[0.93,1.52]$} & $0.69^{* * *}$ & {$[0.52,0.91]$} \\
\hline $\begin{array}{l}\text { La } \\
\text { Réunion }\end{array}$ & $1.38^{* \star \star}$ & {$[1.22,1.57]$} & $1.35^{* * *}$ & {$[1.19,1.55]$} & $1.78^{* * *}$ & {$[1.56,2.03]$} & $1.62^{* * *}$ & {$[1.39,1.89]$} \\
\hline $1929-1945$ & Ref. & & Ref. & & Ref. & & Ref. & \\
\hline 1946-1955 & 0.91 & {$[0.77,1.08]$} & 1.00 & {$[0.82,1.23]$} & 0.93 & {$[0.78,1.12]$} & $0.76^{* * *}$ & {$[0.63,0.91]$} \\
\hline $1956-1965$ & 0.87 & {$[0.73,1.03]$} & 0.93 & {$[0.77,1.12]$} & $0.83^{*}$ & {$[0.69,1.00]$} & $0.49^{* * *}$ & {$[0.41,0.60]$} \\
\hline $1966-1975$ & $0.66^{* * *}$ & {$[0.55,0.79]$} & $0.76^{* *}$ & {$[0.62,0.94]$} & $0.77^{* \star *}$ & {$[0.63,0.94]$} & $0.31^{* \star \star}$ & {$[0.25,0.40]$} \\
\hline Hommes & Ref. & & Ref. & & Ref. & & Ref. & \\
\hline Femmes & $2.07^{* * *}$ & {$[1.63,2.63]$} & $1.79^{* * *}$ & {$[1.45,2.20]$} & $1.64^{* * *}$ & {$[1.36,1.97]$} & $1.54^{* * *}$ & {$[1.24,1.92]$} \\
\hline Migration & Ref. & & Ref. & & Ref. & & Ref. & \\
\hline $\begin{array}{l}\text { Jamais } \\
\text { parti.e }\end{array}$ & $0.25^{* * *}$ & {$[0.20,0.31]$} & $0.76^{*}$ & {$[0.57,1.01]$} & $0.51^{* * *}$ & {$[0.40,0.67]$} & $0.64^{* * *}$ & {$[0.47,0.87]$} \\
\hline $\begin{array}{l}\text { Retour } \\
\text { contraint }\end{array}$ & $0.46^{* * *}$ & {$[0.35,0.60]$} & 1.20 & {$[0.92,1.56]$} & 1.14 & {$[0.89,1.45]$} & 1.16 & {$[0.79,1.69]$} \\
\hline $\begin{array}{l}\text { Retour } \\
\text { par choix }\end{array}$ & $0.43^{* * *}$ & {$[0.34,0.55]$} & $1.36^{* * *}$ & {$[1.10,1.70]$} & $1.25^{* *}$ & {$[1.03,1.51]$} & $1.26^{* *}$ & {$[1.00,1.57]$} \\
\hline $\begin{array}{l}\text { Femmes } \\
\times \text { Jamais } \\
\text { parti·e }\end{array}$ & $0.67^{* *}$ & {$[0.50,0.91]$} & $1.37^{* *}$ & {$[1.00,1.89]$} & 1.04 & {$[0.75,1.45]$} & 0.78 & {$[0.53,1.14]$} \\
\hline $\begin{array}{l}\text { Femmes } \\
\times \text { Retour } \\
\text { contraint }\end{array}$ & $0.44^{* * *}$ & {$[0.27,0.71]$} & 0.74 & {$[0.49,1.13]$} & $0.56^{* * *}$ & {$[0.37,0.84]$} & $0.44^{* * *}$ & {$[0.25,0.78]$} \\
\hline $\begin{array}{l}\text { Femmes } \\
\times \text { Retour } \\
\text { par choix }\end{array}$ & $0.47^{* \star *}$ & {$[0.32,0.67]$} & $0.74^{*}$ & {$[0.55,1.00]$} & $0.50^{* * *}$ & {$[0.37,0.69]$} & $0.49^{* * *}$ & {$[0.35,0.68]$} \\
\hline N & \multicolumn{2}{|c|}{25308} & \multicolumn{2}{|c|}{41055} & \multicolumn{2}{|c|}{40960} & \multicolumn{2}{|c|}{50308} \\
\hline
\end{tabular}


Tableau A2: Résultats des modèles de durée exprimés sous forme d'odds ratios (contrainte)

\begin{tabular}{|c|c|c|c|c|c|c|c|c|}
\hline & \multicolumn{2}{|c|}{$\begin{array}{c}\text { Départ domicile } \\
\text { parental }\end{array}$} & \multicolumn{2}{|c|}{ Premier enfant } & \multicolumn{2}{|c|}{$\begin{array}{c}\text { Première union } \\
\text { cohabitante }\end{array}$} & \multicolumn{2}{|c|}{ Premier mariage } \\
\hline \multicolumn{9}{|c|}{ Âge de 15 (ref.) à 35 ans : coefficients omis } \\
\hline $\begin{array}{l}\text { Bac ou } \\
\text { plus }\end{array}$ & $1.56^{* * *}$ & {$[1.28,1.91]$} & $0.84^{* *}$ & {$[0.72,0.97]$} & $1.37^{* * *}$ & {$[1.18,1.59]$} & $1.84^{* * *}$ & {$[1.56,2.18]$} \\
\hline Brevet & $1.43^{* * *}$ & {$[1.18,1.74]$} & 0.93 & {$[0.77,1.12]$} & $1.27^{* *}$ & {$[1.05,1.53]$} & $1.57^{* * *}$ & {$[1.25,1.97]$} \\
\hline CAP, BEP & $1.17^{*}$ & {$[0.99,1.37]$} & 0.91 & {$[0.79,1.04]$} & 1.04 & {$[0.89,1.21]$} & 1.06 & {$[0.87,1.29]$} \\
\hline $\begin{array}{l}\text { CEP, } \\
\text { aucun }\end{array}$ & Ref. & & Ref. & & Ref. & & Ref. & \\
\hline Étudiant & 0.88 & {$[0.72,1.08]$} & $0.34^{* \star *}$ & {$[0.25,0.45]$} & $0.57^{* * *}$ & {$[0.41,0.80]$} & 0.79 & {$[0.52,1.19]$} \\
\hline $1^{\text {er emploi }}$ & $1.49^{* * *}$ & {$[1.26,1.76]$} & $1.32^{* * *}$ & {$[1.11,1.58]$} & $1.50^{* * *}$ & {$[1.23,1.83]$} & $1.56^{* * *}$ & {$[1.24,1.97]$} \\
\hline $\begin{array}{l}\text { Guade- } \\
\text { loupe }\end{array}$ & Ref. & & Ref. & & Ref. & & Ref. & \\
\hline $\begin{array}{l}\text { Marti- } \\
\text { nique }\end{array}$ & 1.02 & {$[0.89,1.16]$} & 1.01 & {$[0.90,1.14]$} & 1.02 & {$[0.90,1.15]$} & 1.01 & {$[0.88,1.17]$} \\
\hline Guyane & $1.44^{* *}$ & {$[1.05,1.99]$} & $1.55^{* * *}$ & {$[1.25,1.92]$} & 1.18 & {$[0.93,1.52]$} & $0.69^{* * *}$ & {$[0.52,0.91]$} \\
\hline $\begin{array}{l}\text { La } \\
\text { Réunion }\end{array}$ & $1.39^{* * *}$ & {$[1.22,1.58]$} & $1.35^{* * *}$ & {$[1.18,1.54]$} & $1.77^{* * \star}$ & {$[1.55,2.01]$} & $1.61^{* \star \star}$ & {$[1.38,1.88]$} \\
\hline 1929-1945 & Ref. & & Ref. & & Ref. & & Ref. & \\
\hline 1946-1955 & 0.91 & {$[0.77,1.08]$} & 1.00 & {$[0.82,1.23]$} & 0.93 & {$[0.77,1.12]$} & $0.75^{* * *}$ & {$[0.62,0.91]$} \\
\hline $1956-1965$ & 0.87 & {$[0.73,1.03]$} & 0.93 & {$[0.77,1.12]$} & $0.82^{* *}$ & {$[0.68,0.99]$} & $0.49^{* * *}$ & {$[0.41,0.59]$} \\
\hline 1966-1975 & $0.66^{* * *}$ & {$[0.55,0.79]$} & $0.76^{* * *}$ & {$[0.62,0.94]$} & $0.77^{* * *}$ & {$[0.63,0.93]$} & $0.31^{* * *}$ & {$[0.25,0.40]$} \\
\hline Hommes & Ref. & & Ref. & & Ref. & & Ref. & \\
\hline Femmes & $2.07^{* * *}$ & {$[1.63,2.63]$} & $1.79^{* * *}$ & {$[1.45,2.20]$} & $1.63^{* * *}$ & {$[1.35,1.97]$} & $1.54^{* * *}$ & {$[1.24,1.92]$} \\
\hline Migration & Ref. & & Ref. & & Ref. & & Ref. & \\
\hline $\begin{array}{l}\text { Jamais } \\
\text { parti·e }\end{array}$ & $0.25^{* * *}$ & {$[0.20,0.31]$} & $0.76^{*}$ & {$[0.57,1.01]$} & $0.51^{* * *}$ & {$[0.40,0.67]$} & $0.64^{* * *}$ & {$[0.46,0.87]$} \\
\hline $\begin{array}{l}\text { Retour } \\
\text { contraint }\end{array}$ & $0.45^{* * *}$ & {$[0.34,0.58]$} & 1.20 & {$[0.92,1.56]$} & 1.10 & {$[0.84,1.43]$} & 1.10 & {$[0.79,1.52]$} \\
\hline $\begin{array}{l}\text { Retour } \\
\text { par choix }\end{array}$ & $0.44^{* * *}$ & {$[0.34,0.55]$} & $1.37^{* * *}$ & {$[1.10,1.70]$} & $1.26^{* *}$ & {$[1.04,1.52]$} & $1.27^{* *}$ & {$[1.00,1.61]$} \\
\hline $\begin{array}{l}\text { Femmes } \times \\
\text { Jamais } \\
\text { parti·e }\end{array}$ & $0.67^{* *}$ & {$[0.50,0.91]$} & $1.38^{* *}$ & {$[1.00,1.89]$} & 1.04 & {$[0.75,1.45]$} & 0.78 & {$[0.53,1.14]$} \\
\hline $\begin{array}{l}\text { Femmes } \times \\
\text { Retour } \\
\text { contraint }\end{array}$ & $0.46^{* * *}$ & {$[0.28,0.75]$} & 0.85 & {$[0.57,1.25]$} & 0.75 & {$[0.47,1.19]$} & $0.50^{* \star}$ & {$[0.29,0.88]$} \\
\hline $\begin{array}{l}\text { Femmes } \times \\
\text { Retour } \\
\text { par choix }\end{array}$ & $0.46^{* * *}$ & {$[0.31,0.67]$} & $0.71^{* *}$ & {$[0.53,0.97]$} & $0.46^{* * *}$ & {$[0.34,0.62]$} & $0.47^{* * *}$ & {$[0.34,0.65]$} \\
\hline$N$ & \multicolumn{2}{|c|}{25308} & \multicolumn{2}{|c|}{41055} & \multicolumn{2}{|c|}{40960} & \multicolumn{2}{|c|}{50308} \\
\hline
\end{tabular}


Tableau A3 : Résultats des modèles de panel à effets fixes exprimés sous forme d'odds ratios

\begin{tabular}{|c|c|c|c|c|c|c|c|c|}
\hline \multirow[b]{3}{*}{ Âge } & \multicolumn{4}{|c|}{ Retour contraint[1] ou par choix[0] } & \multicolumn{4}{|c|}{ Retour avec[1] ou sans difficultés[0] } \\
\hline & \multicolumn{2}{|c|}{ Mariage } & \multicolumn{2}{|c|}{ Cohabitation } & \multicolumn{2}{|c|}{ Mariage } & \multicolumn{2}{|c|}{ Cohabitation } \\
\hline & $1.59^{\star \star \star}$ & {$[1.57,1.61]$} & $1.41^{* * *}$ & {$[1.40,1.43]$} & $1.59^{* * *}$ & {$[1.57,1.62]$} & $1.41^{* * *}$ & {$[1.40,1.43]$} \\
\hline $\begin{array}{l}\text { Fin } \\
\text { études }\end{array}$ & $8.96^{\star \star *}$ & {$[5.61,14 \cdot 30]$} & $4.28^{* \star *}$ & {$[3.38,5.42]$} & $8.90^{* * *}$ & {$[5.58,14.21]$} & $4 \cdot 32^{* * *}$ & {$[3.41,5.48]$} \\
\hline $\begin{array}{l}\text { Premier } \\
\text { emploi }\end{array}$ & $1.40^{* * *}$ & {$[1.12,1.74]$} & $2.21^{* \star \star}$ & {$[1.90,2.57]$} & $1.38^{* * *}$ & {$[1.11,1.72]$} & $2.19^{* * *}$ & {$[1.88,2.54]$} \\
\hline \multicolumn{9}{|l|}{$\begin{array}{l}\text { En } \\
\text { migration }\end{array}$} \\
\hline $\begin{array}{l}\text { Homme } \\
\times \text { Jamais } \\
\text { parti }\end{array}$ & $0.14^{* * *}$ & {$[0.09,0.23]$} & $0.15^{* * *}$ & {$[0.12,0.20]$} & $0.14^{* * *}$ & {$[0.09,0.23]$} & $0.15^{* * *}$ & {$[0.12,0.20]$} \\
\hline $\begin{array}{l}\text { Homme } \\
\times \\
\text { Retour[1] }\end{array}$ & $3.74^{* * *}$ & {$[1.93,7.23]$} & $1.82^{* * *}$ & {$[1.28,2.60]$} & $3.01^{* * *}$ & {$[1.63,5 \cdot 56]$} & $1.62^{* * *}$ & {$[1.17,2.25]$} \\
\hline $\begin{array}{l}\text { Homme } \\
\times \\
\text { Retour[0] }\end{array}$ & $5 \cdot 32^{* \star *}$ & {$[3.78,7.50]$} & $3.09^{* * *}$ & {$[2.53,3.77]$} & $5.60^{* * *}$ & {$[3.95,7.95]$} & $3.27^{* * *}$ & {$[2.67,4.02]$} \\
\hline $\begin{array}{l}\text { Femme } \times \\
\text { Jamais } \\
\text { partie }\end{array}$ & $0.46^{* \star *}$ & {$[0.36,0.60]$} & $0.41^{* * \star}$ & {$[0.34,0.49]$} & $0.50^{\star * *}$ & {$[0.39,0.64]$} & $0.42^{* * *}$ & {$[0.36,0.51]$} \\
\hline $\begin{array}{l}\text { Femme } \times \\
\text { Retour[1] }\end{array}$ & $0.12^{* * *}$ & {$[0.08,0.16]$} & $0.09^{* * *}$ & {$[0.07,0.12]$} & $0.09^{* * *}$ & {$[0.07,0.13]$} & $0.11^{* * *}$ & {$[0.09,0.14]$} \\
\hline $\begin{array}{l}\text { Femme } \times \\
\text { Retour[o] }\end{array}$ & $0.58^{* * *}$ & {$[0.44,0.76]$} & $0.42^{* * *}$ & {$[0.35,0.50]$} & 0.83 & {$[0.62,1.11]$} & $0.41^{* \star \star}$ & {$[0.34,0.50]$} \\
\hline N & \multicolumn{2}{|c|}{33873} & \multicolumn{2}{|c|}{49182} & \multicolumn{2}{|c|}{33873} & \multicolumn{2}{|c|}{49182} \\
\hline
\end{tabular}

